\title{
Iron Status in Children With Autism Spectrum Disorder
}

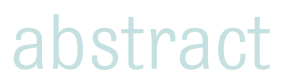

BACKGROUND AND OBJECTIVES: Children with autism spectrum disorders (ASDs) often have food selectivity and restricted diets, putting them at risk for nutritional deficiencies. Previous studies have demonstrated a high prevalence of iron deficiency (ID) in children with ASDs living in Wales, Canada, and Turkey. The objectives of this study were to determine the prevalence of ID and the adequacy of iron intake in children with ASD in the United States.

METHODS: Participants (age 2-11 years recruited from the Autism Treatment Network Diet and Nutrition Study) completed a 3-day diet record $(n=368)$ and had laboratory measures of serum ferritin (SF), complete blood count, iron, total iron binding capacity, and transferrin saturation (TS) $(n=222)$.

RESULTS: Of the 222 participants with laboratory data, 18 (8\%) had SF $<12 \mu \mathrm{g} / \mathrm{L}$ and $2(1 \%)$ had ID defined by both low SF and TS ( 3 children with low SF had missing TS data). One subject had iron deficiency anemia. Fewer than $2 \%$ of subjects had iron intake below the estimated average requirement.

CONCLUSIONS: Although the determination of iron status is complex, these data do not support previous reports that children with ASD are at greater risk for ID than the general population; however, 8\% percent of the sample did demonstrate low SF despite $<2 \%$ of the sample demonstrating iron intake below the estimated average requirement. The prevalence of low SF may be an underestimate, because SF is an acute phase reactant and the study included no measure of inflammation. Pediatrics 2012;130:S154-S159
AUTHORS: Ann Reynolds, MD, ${ }^{a}$ Nancy F. Krebs, MD, ${ }^{a}$

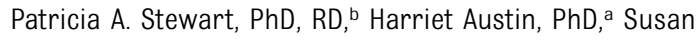
L. Johnson, PhD, ${ }^{\text {a }}$ Nikki Withrow, RD, ${ }^{\text {a }}$ Cynthia Molloy, MD, ${ }^{c}$ S. Jill James, PhD, ${ }^{d}$ Cynthia Johnson, PhD, ${ }^{\text {e Traci Clemons, }}$ PhD, ${ }^{f}$ Brianne Schmidt, RD, ${ }^{b}$ and Susan L. Hyman, MD ${ }^{\mathrm{b}}$

aDepartment of Pediatrics, University of Colorado, Denver, Colorado; ${ }^{b}$ Department of Pediatrics, University of Rochester,

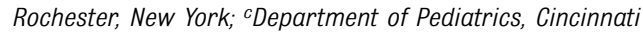
Children's Hospital Medical Center, Cincinnati, Ohio; ${ }^{d}$ Department of Pediatrics, University of Arkansas, Little Rock, Arkansas; ${ }^{e}$ Department of Pediatrics, University of Pittsburgh, Pittsburgh, Pennsy/vania; and ${ }^{f}$ EMMES Corporation, Rockville, Maryland

KEY WORDS

autism, iron, ferritin, nutrition

\section{ABBREVIATIONS}

ASD-autism spectrum disorder

ATN-Autism Treatment Network

EAR-estimated average requirement

Hgb-hemoglobin

ID-iron deficiency

IDA-iron deficiency anemia

SF-serum ferritin

TS-transferrin saturation

This manuscript has been read and approved by all authors.

This paper is unique and not under consideration by any other publication and has not been published elsewhere.

www.pediatrics.org/cgi/doi/10.1542/peds.2012-0900M

doi:10.1542/peds.2012-0900M

Accepted for publication Aug 8, 2012

Address correspondence to Ann Reynolds, MD, Children's Hospital Colorado, 13123 East 16th Avenue, B-140, Aurora, Colorado 80045. E-mail: ann.reynolds@childrenscolorado.org

PEDIATRICS (ISSN Numbers: Print, 0031-4005; Online, 1098-4275). Copyright (C) 2012 by the American Academy of Pediatrics FINANCIAL DISCLOSURE: The authors have indicated they have no financial relationships relevant to this article to disclose. 
Autism spectrum disorders (ASDs) are a group of disorders defined by dysfunction in 3 areas of development: social interaction, communication, and restricted interests and repetitive behaviors. The third area of symptoms is often associated with a need for sameness, which is thought to contribute to feeding issues, such as mealtime rituals and a limited food repertoire ${ }^{1,2}$ reported in $70 \%$ to $90 \%$ of children with ASD. ${ }^{3-5}$ Previous studies have raised concerns that intake of iron, $B$ vitamins, vitamin $D$, vitamin $\mathrm{K}$, calcium, and zinc may be deficient in up to one-third of children with ASDs, and that dietary insufficiency may be more common in children on a restrictive diet ${ }^{6-9}$; however, the Autism Treatment Network (ATN) Diet and Nutrition Study, also published in this supplement, ${ }^{10}$ did not find differences. Indeed, the prevalence of inadequate intake was similar to the general pediatric population. Three studies have identified concerns regarding iron status in children with ASDs. In South Wales, Turkey, and Canada, low serum ferritin (SF) was found in $7 \%$ to $52 \%$ of children with ASDs by using SF of $<10$ to $12 \mu \mathrm{g} / \mathrm{L}$ to define a low value. Anemia was found in $1 \%$ to $15 \%$ of children with ASDs and varied by country and type of ASD diagnosis. It was not always possible to determine which children with anemia had iron deficiency anemia (IDA) in these studies. ${ }^{11-13}$ Ferritin alone was used to evaluate iron deficiency (ID). The diagnosis of ID is quite challenging, ${ }^{14}$ and the regulation of iron absorption is complex. ${ }^{15,16}$ This complexity requires multiple measures of iron status to determine iron sufficiency. Although clinicians typically use hemoglobin (Hgb), a measure of anemia, as a way to monitor iron status, iron stores become deficient before a decrease in Hgb. Transferrin saturation (TS) and SF are widely used to assess iron status. Previous studies in children with ASD did not use multiple markers to make a diagnosis of ID.
Iron intake is not always associated with iron status. Iron is consumed from dietary sources and very low intake leads to deficiency; however, absorption can vary greatly in those with sufficient intake. For example, the bioavailability of iron from nonheme sources may be less than from heme sources; vitamin $C$ can enhance absorption; calcium, divalent cations, and other substances present in food can inhibit absorption; and poor absorption may be associated with chronic inflammation. ${ }^{17}$ These variations lead to difficulty in determining iron requirements for a population, because individuals need different amounts of dietary iron based on the sources of iron and their rates of absorption. Only 1 study has compared iron intake with iron status in a small number of children with ASD and restless sleep. ${ }^{7}$ The study found SF was not associated with iron intake (estimated from 3-day food record); however, SF levels increased after supplementation with iron, suggesting that increasing intake improved iron status. No study has compared iron intake with laboratory measures of iron sufficiency in a large group of children with ASD. The association between iron intake and iron status is important to evaluate because of concerns that poor absorption may play a role in ID in children with adequate intake.

Despite concerns about dietary inadequacy and evidence that ID can affect attention, sleep, and cognitive development, ${ }^{18-24}$ no studies in the United States have examined iron status or the relationship between iron intake and iron status in children with ASD. It is important to identify remediable medical risk factors for both behavioral and medical disorders in children with ASD. This study was undertaken to determine if children with ASD have a high prevalence of ID, IDA, or insufficient iron intake, and the relationship of iron intake to iron status.

\section{METHODS}

\section{Sample}

Our study used a cross-sectional, observational design to study iron status and iron intake in a geographically diverse population of children with ASD. Subjects were recruited from 5 sites within the ATN (Rochester, NY; Denver, CO; Cincinnati, OH; Pittsburgh, PA; and Little Rock, AR). Participants were 2 to 11 years of age at enrollment and had all received a diagnosis of autism, pervasive developmental disorder (not otherwise specified), or Asperger syndrome by Diagnostic and Statistical Manual of Mental Disorders, Fourth Edition criteria $^{25}$ and the Autism Diagnostic Observation Schedule. ${ }^{26}$ Cognitive testing from clinical evaluations is available from the ATN registry. Parents completed a demographic questionnaire including education and family income, allowing for determination of socioeconomic status using the Hollingshead Four Factor Index score. ${ }^{27}$ This study included participants from a larger study of diet and nutrition, which included collection of dietary measures. ${ }^{10}$ Institutional review board approval was obtained at all 5 sites and appropriate consent and assent was obtained for all subjects.

\section{Blood Specimen Collection}

Laboratory measures were collected on the day of the diet record training and were optional. Sixty percent of families consented to a blood draw, which was often paired with other needed clinical laboratories. Topical lidocaine/prilocaine cream was offered for application 20 minutes before the blood draw. Blood was sent for complete blood count, SF, TS, iron, and total iron-binding capacity. Specimen collection and analysis were performed at each site, often by the local Clinical and Translational Research Center.

\section{SF}

SF lacks a uniform reference range in the literature. Because of variability in 
cut points for low values between sites, a cutoff of $<12 \mu \mathrm{g} / \mathrm{L}$ was used as suggested by the World Health Organization. ${ }^{28}$ The clinical laboratories across sites used different commercial methods for analysis of ferritin: Colorado, Siemens (Dade-Behring) BNII Nepholometric Ferritin Assay; Rochester, ElectroChemiluminescence immunoassay made by Roche; Cincinnati, Siemens Dimension RxL; Pittsburgh, Beckman Coulter; Arkansas, Enhanced Chemiluminescense assay, $\mathrm{J}$ and $\mathrm{J}$ Ortho Clinical Diagnostics.

\section{TS}

TS is a calculated value based on iron divided by total iron-binding capacity. ATS of $<10 \%$ was considered abnormal. ${ }^{29}$

\section{IDA}

Anemia is not specific for ID, and therefore some measure of iron status is also needed to determine that anemia is attributable to ID. Low SF along with anemia is often used to help make a diagnosis of IDA. For this study, IDA was defined as low Hgb $(<11.0 \mathrm{~g} / \mathrm{dL}$ for children $<5$, and $<12.0 \mathrm{~g} / \mathrm{dL}$ for children $6-11)^{29}$ and SF $<12 \mu \mathrm{g} / \mathrm{L}{ }^{28}$

\section{Ferritin Model of ID}

NHANES has reported ID based on the "ferritin model" (2 of 3 measures low: SF, TS, and erythrocyte protoporphyrin). ${ }^{30}$ Transferrin saturation is widely used to evaluate ID, but because of diurnal variation in serum iron requires a second measure. SF is considered to be a good indicator of low iron stores but is also an acute phase reactant and can be falsely elevated in the face of acute or chronic inflammation. ${ }^{14}$ Because erythrocyte protoporphyrin is not widely used, we defined ID based on low SF and low TS.

\section{Diet Records}

Food records were collected for each child over 3 consecutive days, including 1 weekend day and included dietary supplements. The methods for collection and analysis of diet diary data are described in the accompanying article on nutrition. ${ }^{10}$ The estimated average requirement (EAR) is the average daily nutrient intake level estimated to meet the requirements of half of the healthy individuals in a group. It is used only for nutrients for which a daily recommended intake has been established. Iron intake was determined from dietary and supplemental sources. Nutrient intake was adjusted for day-to-day variability. The cut point method, which reflects the proportion of individuals within a group with inadequate intake, was used to determine the percentage of individuals with intakes below the EAR. ${ }^{31}$ Because of its skewed requirement distribution, the full probability approach was used to determine the prevalence of insufficient iron intake.32

\section{Analysis}

A 2-sample $t$ test for continuous measures and $\chi^{2}$ tests for categorical variables were used to examine differences between the participants in the larger diet and nutrition study whose parents consented to blood testing and those who did not. Descriptive statistics, including the mean, SD, and median values for SF and TS were computed for the sample. In addition, the percentage of these values reported to be below or within the normal range were computed for the total sample for these laboratory tests. Exact $\chi^{2}$ tests (proportion) and analysis of variance tests (means) were used to determine differences among sites in measures of ID. The method used to determine percentage below the EAR was a group distribution of the percentage below the EAR cutoff. All analyses were conducted by using SAS version 9.2 (SAS Institute Inc, Cary, NC).

\section{RESULTS}

Of 368 subjects enrolled in the ATN Diet and Nutrition Study, 222 (60\%) had blood drawn for iron studies. Ofthese, 162 (73\%) were diagnosed with autism, 40 (18\%) with pervasive developmental disordernot otherwise specified, and 20 (9\%) with Asperger syndrome. The mean age of children who had blood specimens collected was 5.3 years, range 2 to 11 years, and $87 \%$ were male. No significant differences were noted in those who consented to blood specimens based on race/ethnicity, age, cognitive function, socioeconomic status, or iron intake as estimated from 3-day food records (Table 1). There was significant variability in the percentage of individuals consenting to blood draw (32\% to $88 \%, P<$ .001) across the 5 participating sites. However, there was no difference by site in prevalence of $\mathrm{SF}<12 \mu g / \mathrm{L}(P=.19)$ or in mean SF $(P=.13)$. There was also no difference by site in prevalence of TS $<10 \%(P=.95)$ or in mean TS $(P=.20)$. SF $<12 \mu \mathrm{g} / \mathrm{L}$ was present in $8 \%$ of participants, indicating low iron stores. Only 2 children ( $1 \%$ of the sample) met our criteria for ID. Unfortunately, 3 of the 18 children with low SF had missing data for TS. One child in the sample met criteria for IDA (Table 2). ID rates varied by age group in the NHANES study (1999-2000): 7\% of 1- to 2-year-olds, $5 \%$ of 3- to 5 -year-olds, and $4 \%$ of 6 - to 11-year-olds after excluding those with elevated C-reactive protein. ${ }^{33}$ Our data found lower rates of ID in all age groups compared with NHANES data. The prevalence of low SF alone in the NHANES sample is not published. In our sample, the percentage of children with intakes less than EAR was $<2 \%$ and increased with age. In contrast, low SF was more common in the younger group.

\section{DISCUSSION}

This is the first study to evaluate iron status in a large, well-characterized population of children with ASD in the United States. Unlike previous studies in children with ASD, this study included measurement of both SF and TS to 
TABLE 1 Characteristics of Study Participants With and Without Laboratory Data

\begin{tabular}{lccc}
\hline \multicolumn{1}{c}{ Characteristic } & No Laboratory Data $n(\%)$ & Laboratory Data $n$ (\%) & $P$ Value \\
\hline Race $(n=368)$ & & & .74 \\
$\quad$ Asian & $3(1)$ & $4(2)$ & \\
African American & $6(4)$ & $12(5)$ & \\
$\quad$ Native American & $0(0)$ & $1(0.5)$ & \\
$\quad$ White & $130(89)$ & $188(85)$ & \\
$\quad$ Mixed race & $7(5)$ & $17(8)$ & .02 \\
ASD Diagnosis $(n=368)$ & & & \\
$\quad$ Autism & $92(63)$ & $162(73)$ & .37 \\
Asperger's & $9(6)$ & $20(9)$ & .39 \\
$\quad$ PDD/NOS & $45(31)$ & $193(18)$ & .84 \\
Gender, male $(n=368)$ & $122(83)$ & $5.37(2.50)$ & .50 \\
Age, $\mathrm{y}(n=368)$ & $5.15(2.31)$ & $80.43(22.87)$ & .83 \\
IQ score, mean (SD) $(n=307)$ & $80.97(23.62)$ & $44.45(12.97)$ & \\
Hollingshead score $(n=339)$ & $43.48(13.19)$ & $12.40 \mathrm{mg}(11.56)$ & \\
Intake of dietary iron $(n=292)$ & $12.61 \mathrm{mg}(10.56)$ & & \\
\hline
\end{tabular}

PDD/NOS, pervasive developmental disorder-not otherwise specified.

identify ID. This is important because SF is an acute phase reactant and elevated in the face of inflammation. Inflammation would artificially increase the ferritin levels and decrease suspicion of ID. The use of both SF and TS was included because of the lack of sensitivity or specificity for each test alone. ${ }^{34}$ This is also the first large study to compare iron status to dietary iron intake from both food and supplements. The laboratory findings do not support previous studies from Canada, South Wales, and Turkey that reported higher rates of ID/low SF in children with ASD than in the general population. ${ }^{11-13}$ Decreasing rates of ID in the United States over the past decades $^{29}$ may explain some of the differences between the findings of this study and older studies in children with ASD.

TABLE 2 Markers of Iron Status in Children With ASD

\begin{tabular}{lcc}
\hline \multicolumn{1}{c}{ Marker } & $\begin{array}{c}\text { Total No. With } \\
\text { Available Data }\end{array}$ & $\begin{array}{c}\text { No. With Low } \\
\text { Levels (\%) }\end{array}$ \\
\hline Low SF $(<12 \mu \mathrm{g} / \mathrm{L})$ & 218 & $18(8)$ \\
Low TS $(<10 \%)$ & 199 & $12(6)$ \\
Iron deficiency (Iow & 195 & $2(1)$ \\
$\quad$ SF and TS) & & $7(3)$ \\
Anemiab & 219 & $1(<1)$ \\
ID anemia (anemia & 213 & \\
$\quad$ and low ferritin) & & \\
\hline
\end{tabular}

Some children had missing laboratory data because of difficulty drawing an adequate volume of blood.

a Three children with SF $<12$ did not have TS levels drawn. ${ }^{\mathrm{b}}$ Anemia $=\mathrm{Hgb}<11.0 \mathrm{~g} / \mathrm{dL}$ for children $<5$ and $<12.0 \mathrm{~g} / \mathrm{dL}$ for children 6 to 11 .
Identifying ID when present is important, because ID, even without anemia, has adverse consequences on developmental and behavioral function in infants $^{35}$ and children. ${ }^{36,37}$ Iron plays an important role in dopamine metabolism in the brain ${ }^{38}$ and is a cofactor for tyrosine hydroxylase responsible for dopamine synthesis. ${ }^{39}$ Inattention and working memory have been reported to be negatively impacted by ID in children with typical development, ${ }^{21}$ with similar findings in animal models. ${ }^{40}$ Several authors have found low SF/ID in children with attention-deficit/ hyperactivity disorder. ${ }^{18,20,37,41,42}$ This is not a uniform finding, however. ${ }^{19}$

This study has several limitations. Although our data found $8 \%$ of children had low iron stores (SF $<12 \mathrm{ng} / \mathrm{mL}$ ), we did not have a marker of inflammation and may have missed some cases of ID owing to falsely elevated SF. Our sites demonstrated variability in cut points for low SF values, necessitating use of a single cutoff of $<12 \mu g / L$, which may have led to under identification of low SF. Unfortunately, 3 children with low SF did not have a TS drawn because of difficulty obtaining an adequate quantity of blood. The prevalence of ID was only $1 \%$ but may have been as high as $2.5 \%$. Although the prevalence of ID appeared lower than previous NHANES data, the most recent available NHANES data are 10 years old. ${ }^{33} \mathrm{It}$ is possible that the prevalence of ID in the general population may have decreased since that time. The sample of children with ASD consisted of volunteers recruited from a research registry and may not be representative of all children with ASD. We also excluded monolingual Spanish speakers because of constraints regarding diagnosis, and this procedure may have excluded a group particularly prone to ID.43 Families who had more concerns about their child's nutritional status might have been more likely to consent for the optional laboratory testing. This may have been minimized, however, as the blood test was often performed at the same time as the initial etiologic testing recommended at diagnosis, such as chromosomal microarray. Indeed, the prevalence of dietary insufficiency in children with ASD was similar to that of the general pediatric population represented in NHANES healthy subjects..$^{10}$ When the appropriate evaluation metric for comparison, the EAR, was used, children with ASD did not differ from the NHANES population in iron intake. Of note, major sources of iron in the diet of these children were from enriched or fortified foods, such as breakfast cereals. Nonheme sources of iron may be less available for absorption than heme (meat)-based sources. ${ }^{17} \mathrm{Be}-$ cause of the skewed requirement distribution, the EAR for iron can be used only to do group analysis. Individual analysis is not possible.

\section{CONCLUSIONS}

Although determination of iron status is complex, children with ASD are at risk for low SF, which has been associated with attention and sleep problems. ${ }^{18-24}$ Dietary inadequacy of iron does not appear to be the only risk factor for low iron stores. Future studies will need to explore the complexity of iron metabolism and include central laboratory testing, markers of inflammation such 
as C-reactive protein, and other markers of iron absorption. Biomarkers such as hepcidin and transferrin receptor have been found to play an important role in iron absorption and transport into red blood cells. ${ }^{16}$ These tests are promising but not widely available, and normal values for children have not been established. Iron supplementation of children with ASD is not recommended without documentation of

\section{REFERENCES}

1. Schreck KA, Williams K, Smith AF. A comparison of eating behaviors between children with and without autism. J Autism Dev Disord. 2004;34(4):433-438

2. Valicenti-McDermott M, McVicar K, Rapin I, Wershil BK, Cohen H, Shinnar S. Frequency of gastrointestinal symptoms in children with autistic spectrum disorders and association with family history of autoimmune disease. J Dev Behav Pediatr. 2006;27 (suppl 2):S128-S136

3. Ahearn WH, Castine T, Nault K, Green G. An assessment of food acceptance in children with autism or pervasive developmental disorder-not otherwise specified. J Autism Dev Disord. 2001;31(5):505-511

4. Nieminen-von Wendt T, Paavonen JE, Ylisaukko$0 j a \mathrm{~T}$, et al. Subjective face recognition difficulties, aberrant sensibility, sleeping disturbances and aberrant eating habits in families with Asperger syndrome. BMC Psychiatry. 2005;5:20

5. Schreck KA, Williams K. Food preferences and factors influencing food selectivity for children with autism spectrum disorders. Res Dev Disabil. 2006;27(4):353-363

6. Cornish E. Gluten and casein free diets in autism: a study of the effects on food choice and nutrition. J Hum Nutr Diet. 2002; 15(4):261-269

7. Dosman CF, Brian JA, Drmic IE, et al. Children with autism: effect of iron supplementation on sleep and ferritin. Pediatr Neurol. 2007;36(3):152-158

8. Herndon AC, DiGuiseppi C, Johnson SL, Leiferman J, Reynolds A. Does nutritional intake differ between children with autism spectrum disorders and children with typical development? J Autism Dev Disord. 2009;39(2):212-222

9. Lindsay RL, Eugene Arnold L, Aman MG, et al. Dietary status and impact of risperidone on nutritional balance in children parameters of iron status, and treatment should be monitored for laboratory response. Iron treatment can result in side effects, including constipation and gastrointestinal distress, ${ }^{44-46}$ and there is risk of toxicity with overdose. Given the implications for depleted iron stores to affect sleep, attention, and other medical and behavioral symptoms commonly identified in children with ASD, screening for

with autism: a pilot study. J Intellect Dev Disabil. 2006;31(4):204-209

10. Hyman SL, Stewart P, Schmidt B, et al. Nutrient intake from food in children with autism. Pediatrics. 2012;130 (supp 2): XXXXX

11. Dosman CF, Drmic IE, Brian JA, et al. Ferritin as an indicator of suspected iron deficiency in children with autism spectrum disorder: prevalence of low serum ferritin concentration. Dev Med Child Neurol. 2006;48(12):1008-1009

12. Hergüner $S$, Keleşoğlu FM, Tanıdır C, Cöpür M. Ferritin and iron levels in children with autistic disorder. Eur J Pediatr. 2012;171(1): 143-146

13. Latif A, Heinz P, Cook R. Iron deficiency in autism and Asperger syndrome. Autism. 2002;6(1):103-114

14. Zimmermann MB. Methods to assess iron and iodine status. Br J Nutr. 2008;99(suppl 3):S2-S9

15. Ganz T. Iron homeostasis: fitting the puzzle pieces together. Cell Metab. 2008;7(4):288290

16. Piperno A, Mariani R, Trombini P, Girelli D. Hepcidin modulation in human diseases: from research to clinic. World J Gastroenterol. 2009;15(5):538-551

17. Geissler C, Singh M. Iron, meat and health. Nutrients. 2011;3(3):283-316

18. Konofal E, Cortese S, Marchand M, Mouren MC, Arnulf I, Lecendreux M. Impact of restless legs syndrome and iron deficiency on attention-deficit/hyperactivity disorder in children. Sleep Med. 2007;8(7-8):711-715

19. Millichap JG, Yee MM, Davidson SI. Serum ferritin in children with attention-deficit hyperactivity disorder. Pediatr Neurol. 2006;34(3):200-203

20. Oner P, Dirik EB, Taner Y, Caykoylu A, Anlar 0 . Association between low serum ferritin and restless legs syndrome in patients iron stores should be considered in children with ASD presenting with these symptoms. Although our data indicate low likelihood of dietary insufficiency of iron, obtaining a dietary history is still appropriate.

\section{ACKNOWLEDGMENTS}

The authors acknowledge the members of the Autism Speaks Autism Treatment Network for use of the data. with attention deficit hyperactivity disorder. Tohoku J Exp Med. 2007;213(3):269-276

21. Otero GA, Pliego-Rivero FB, PorcayoMercado R, Mendieta-Alcántara G. Working memory impairment and recovery in iron deficient children. Clin Neurophysiol. 2008; 119(8):1739-1746

22. Picchietti DL, Rajendran RR, Wilson MP, Picchietti MA. Pediatric restless legs syndrome and periodic limb movement disorder: parent-child pairs. Sleep Med. 2009;10 (8):925-931

23. Picchietti MA, Picchietti DL, England SJ, et al. Children show individual night-tonight variability of periodic limb movements in sleep. Sleep. 2009;32(4):530-535

24. Simakajornboon N, Kheirandish-Gozal L, Gozal D. Diagnosis and management of restless legs syndrome in children. Sleep Med Rev. 2009;13(2):149-156

25. American Psychiatric Association. Diagnostic and Statistical Manual of Mental Disorders. 4th ed. Washington, DC: American Psychiatric Association; 1994

26. Lord C, Shulman C, DiLavore P. Regression and word loss in autistic spectrum disorders. J Child Psychol Psychiatry. 2004;45 (5):936-955

27. Hollingshead A. Four factor index of social status. Yale Journal of Sociology. 2011;8:21-51

28. World Health Organization. Assessing the Iron Status of Populations: Report of a Joint World Health Organization/Centers for Disease Control and Prevention Technical Consultation on the Assessment of Iron Status at the Population Level. Geneva, Switzerland: World Health Organization, Centers for Disease Control and Prevention; 2005

29. Baker RD, Greer FR; Committee on Nutrition American Academy of Pediatrics. Diagnosis and prevention of iron deficiency and irondeficiency anemia in infants and young 
children (0-3 years of age). Pediatrics. 2010;126(5):1040-1050

30. Cogswell ME, Looker AC, Pfeiffer CM, et al. Assessment of iron deficiency in US preschool children and nonpregnant females of childbearing age: National Health and Nutrition Examination Survey 2003-2006. Am J Clin Nutr. 2009;89(5):1334-1342

31. Institute of Medicine. Dietary Reference Intakes: Applications and Dietary Assessment. Washington, DC: National Academy Press; 2000

32. Murphy SP, White KK, Park SY, Sharma S. Multivitamin-multimineral supplements' effect on total nutrient intake. Am J Clin Nutr. 2007;85(1):280S-284S

33. Looker A, Cogswell M, Gunter E; Centers for Disease Control and Prevention (CDC). Iron deficiency-United States, 1999-2000. MMWR Morb Mortal Wkly Rep. 2002;51(40):897-899

34. Looker AC, Dallman PR, Carroll MD, Gunter EW, Johnson CL. Prevalence of iron deficiency in the United States. JAMA. 1997; 277(12):973-976

35. Beard JL. Why iron deficiency is important in infant development. J Nutr. 2008;138(12): 2534-2536
36. Halterman JS, Kaczorowski JM, Aligne CA, Auinger P, Szilagyi PG. Iron deficiency and cognitive achievement among school-aged children and adolescents in the United States. Pediatrics. 2001;107(6):1381-1386

37. Konofal E, Lecendreux M, Arnulf I, Mouren MC. Iron deficiency in children with attentiondeficit/hyperactivity disorder. Arch Pediatr Adolesc Med. 2004;158(12):1113-1115

38. Unger EL, Wiesinger JA, Hao L, Beard JL. Dopamine D2 receptor expression is altered by changes in cellular iron levels in PC12 cells and rat brain tissue. J Nutr. 2008;138(12):2487-2494

39. Bianco LE, Wiesinger J, Earley CJ, Jones BC, Beard JL. Iron deficiency alters dopamine uptake and response to L-DOPA injection in Sprague-Dawley rats. J Neurochem. 2008; 106(1):205-215

40. Felt BT, Beard JL, Schallert T, et al. Persistent neurochemical and behavioral abnormalities in adulthood despite early iron supplementation for perinatal iron deficiency anemia in rats. Behav Brain Res. 2006; 171(2):261-270

41. Calarge C, Farmer C, DiSilvestro R, Arnold LE. Serum ferritin and amphetamine response in youth with attention-deficit/hyperactivity disorder. J Child Adolesc Psychopharmacol. 2010;20(6):495-502

42. Cortese S, Konofal E, Bernardina BD, Mouren MC, Lecendreux M. Sleep disturbances and serum ferritin levels in children with attention-deficit/hyperactivity disorder. Eur Child Adolesc Psychiatry. 2009;18(7):393399

43. Brotanek JM, Gosz J, Weitzman M, Flores G. Iron deficiency in early childhood in the United States: risk factors and racial/ ethnic disparities. Pediatrics. 2007;120(3): 568-575

44. Coplin M, Schuette S, Leichtmann G, Lashner B. Tolerability of iron: a comparison of bis-glycino iron II and ferrous sulfate. Clin Ther. 1991;13(5):606-612

45. Frykman E, Bystrom M, Jansson U, Edberg A, Hansen T. Side effects of iron supplements in blood donors: superior tolerance of heme iron. J Lab Clin Med. 1994;123(4): 561-564

46. Rimon E, Kagansky N, Kagansky M, et al. Are we giving too much iron? Low-dose iron therapy is effective in octogenarians. $A m ~ J$ Med. 2005;118(10):1142-1147 